\title{
Size-effects in time-dependent mechanics in metallic MEMS
}

\author{
L.I.J.C. Bergers ${ }^{1,2,3, a}$, N.K.R. Delhey ${ }^{1}$, J.P.M. Hoefnagels ${ }^{1}$, and M.G.D.Geers ${ }^{1}$ \\ 1 Eindhoven University of Technology, Dep. of Mechanical Engineering, P.O. Box 513, 5600 MB, \\ Eindhoven, The Netherlands \\ 2 Foundation for Fundamental Research on Matter, P.O. Box 3021, 3502 GA, Utrecht, The Nether- \\ lands \\ 3 Materials Innovation Institute, P.O. Box 5008, 2600 GA, Delft, The Netherlands
}

\section{Summary}

Reliability of microelectromechanical systems (MEMS) depends a.o. on time-dependent deformation such as creep and fatigue [1]. It is known from literature that this behavior is affected by size-effects: the interaction between microstructural length scales and dimensional length scales [2,3]. Not much research has focused on characterizing size-effects in time-dependent material behavior, specifically for free-standing thin films [3]. This study investigates size-effects caused by grain statistics in timedependent deformation in $\mu \mathrm{m}$-sized free-standing aluminum cantilever beams.

A numeric-experimental method is used to determine material parameters. The experiment entails applying a constant deflection to the micro-beams for a prolonged period. The deflection is achieved with $50 \mathrm{~nm}$ resolution via a micro-clamp. The beams are then released. Immediately the deformation evolution is recorded by acquiring surface height profiles with a confocal optical profiler. Image correlation of the full-field beam profiles is applied to correct for specimen drift and tilt. The experiment yields the tip deflection as function of time with $\sim 3 \mathrm{~nm}$ precision. In the numerical part, this data is combined with a finite element model based on a standard-solid material model. In this way material parameters describing time-dependent behavior are extracted. The time constant for the deflection evolution is determined within $20 \%$, as verified by predicting a different experiment. Figure 1 shows the model and the numeric prediction of an experiment.

To investigate the size-effects of grain statistics, orientation imaging microscopy (OIM) is employed directly on the free-standing cantilevers, see figure 2 . This work correlates the obtained timedependent material parameters to the actual grain sizes, grain boundary length and texture orientation per specimen. Insights into the interplay between micro-mechanism and grain characteristic and the effect on the time-dependent material behavior are presented.

\section{References}

1. W.M. van Spengen, Microelectronics Reliability 43, (2003) 1049-1060

2. G. Dehm, C. Motz, et.al., Advanced Engineering Materials 8 (2006) 1033-1045

3. T. Connolley, P. E. McHugh, M. Bruzzi, Fatigue Fract. Eng Mater. Struct. 28, (2005) 1119-1152

a e-mail: 1.i.j.c.bergers@tue.nl

This is an Open Access article distributed under the terms of the Creative Commons Attribution-Noncommercial License 3.0, which permits unrestricted use, distribution, and reproduction in any noncommercial medium, provided the original work is properly cited. 

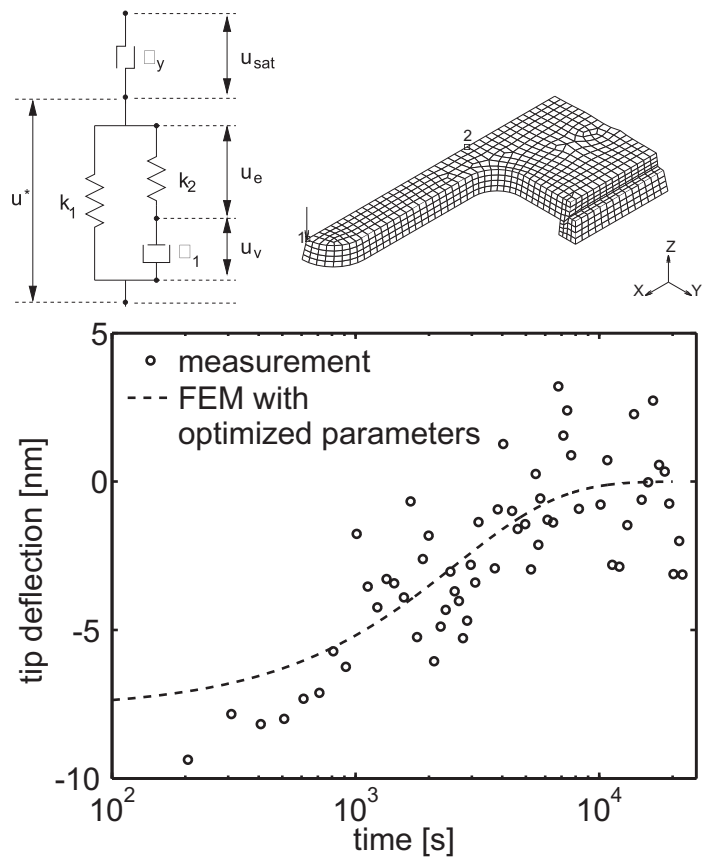

Fig. 1. Numeric-experimental method to obtain material parameters from a time-dependent behavior measurement.
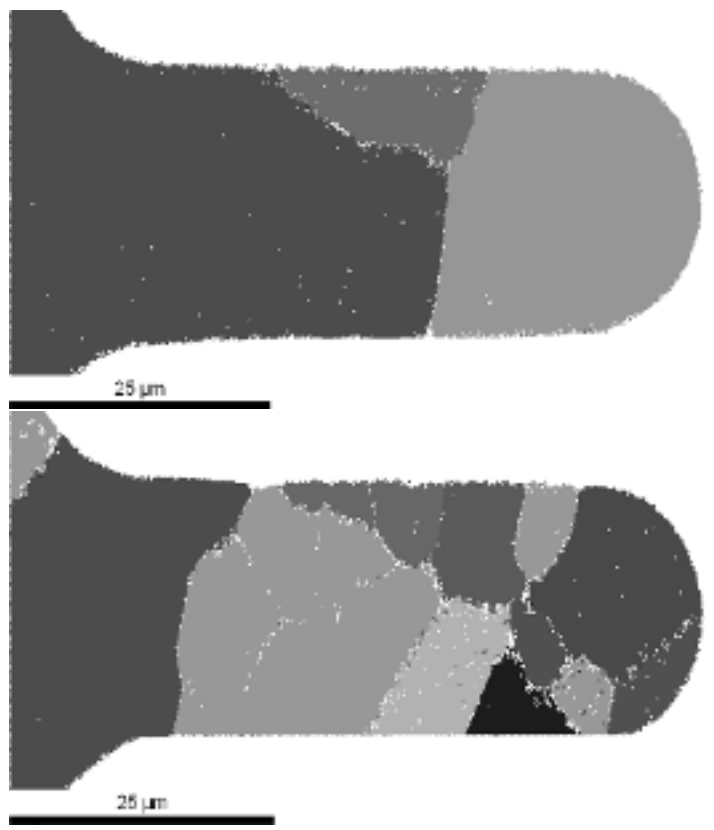

Fig. 2. Grains in cantilever beams visualized by OIM for a specimen containing (t) few grains and (b) many grains. 